\title{
PLSA BASED TOPIC MIXTURE LANGUAGE MODELING APPROACH
}

\author{
Shuanhu Bai and Haizhou Li \\ Institute for Infocomm Research, 1 Fusionopolis Way \#21-01 Connexis, Singapore 138632 \\ \{sbai,hli\}@i2r.a-star.edu.sg
}

\begin{abstract}
In this paper, we propose a method to extend the use of latent topics into higher order n-gram models. In training, the parameters of higher order n-gram models are estimated using discounted average counts derived from the application of probabilistic latent semantic analysis(PLSA) models on n-gram counts in training corpus. In decoding, a simple yet efficient topic prediction method is introduced to predict its topic given a new document. The proposed topic mixture language model (TMLM) displays two advantages over previous methods: 1) having the ability of building topic mixture n-gram LM $(\mathrm{n}>1)$ and, 2) without requiring a big general baseline LM. The experimental results show that TMLMs, even using smaller number of topics, outperform LMs implemented using both standard n-gram approach and unsupervised adaptation approaches in terms of perplexity reductions.
\end{abstract}

Index Terms - language modeling, topic mixture language model, PLSA

\section{INTRODUCTION}

This paper proposes a topic mixture language modeling approach by extending the use of latent topic information of PLSA models into higher order n-gram models. The proposed method achieves better performance compared with both standard n-gram modeling approach and unsupervised adaptation approach.

Inspired by the concept of mixture models, the application of topic mixtures in language modeling tasks can be found decades ago. In their early efforts, manually labeled data is used for training and naive Bayesian text classification method for decoding [6]. An attempt with unsupervised method was made by [7] in which topic decomposition were achieved by vector clustering and Expectation Maximization (EM) algorithms. Another unsupervised approach is introduced in by [4]. The proposed technology of latent semantic analysis (LSA) is implemented by using singular value decomposition algorithm, and significant improvements reported in terms of both perplexity and word error rate reductions.

With the progresses made in document modeling technologies [9] recently, attentions have been attracted to incorporate the results into language modeling tasks
[1][2][3]. The main focuses of these efforts are in the area of unsupervised LM adaptation based on PLSA framework. A common practice they share is to interpolate a document model with a general purpose baseline model.

We investigate topic mixture language modeling approach from different prospects. The basic idea of our approach is to make use of latent topic information derived from PLSA in a different way by extending mixture-of-unigram to mixture-of-n-gram.

The paper is organized as follows. In section 2 we give some brief introduction of PLSA approach. The method of building higher order mixture components will be described in Section 3. We introduce Bayesian topic prediction method in 4. Section 5 will be dedicated to various experiments on perplexity tests with LDC Gigabyte English corpus. Section 6 will conclude with discussions of our findings and possible issues that need further studies.

\section{PLSA FRAMEWORK}

Under the formalism of PLSA[1][9], the probability of a word in a document is described as:

$$
p_{P L S A}(w)=\sum_{t} p(t \mid d) p(w \mid t)
$$

where both $p(t \mid d)$ and $p(w \mid t)$ can be obtained by applying EM algorithm on the likelihood of a collection of documents. Here $p(t \mid d)$ represents the result of topic decomposition (TD) of the document collection, it can also be interpreted as the soft classification of documents into $t$ latent topics, or portion of topics of the documents belonging to; while $p(w \mid t)$ represents the overall word distributions over topics, it can also be interpreted as the mixture components of the mixture model combined together by the portion of topics. To obtain such values, the first step is to build a word by document table where each element $C(w, d)$ is the count of a word $w$ in the corresponding document $d$. PLSA essentially provide solutions for expressing documents in the form of bag-ofwords into fixed number of topic mixture-of-unigrams by the process of TD. The EM procedure for estimating both $p(t \mid d)$ and $p(w \mid t)$ takes place by setting both of the parameters as random probability values first, and iterates 
the E-step indicated by Eq.(2) and M-step indicated by Eq.(3) and Eq.(4) until convergence.

E-step:

$$
\begin{aligned}
& p(t \mid d, w)=\frac{p(t \mid d) p(w \mid t)}{\sum_{t} p(t \mid d) p(w \mid t)} \\
& \text { M-step: } \\
& p(w \mid t)=\frac{\sum_{d} C(w, d) p(t \mid d, w)}{\sum_{w} \sum_{d} C(w, d) p(t \mid d, w)} \\
& p(t \mid d)=\sum_{w} C(w, d) p(t \mid d, w) / C(d)
\end{aligned}
$$

The meaning of Eq.(2) can be interpreted as the probability of topic $t$ is used in documents $d$ for generating word $w$. Subsequently the item of $C(w, d) p(t \mid d, w)$ in (3) and (4) can be explained as the expected counts of $t$ is used in documents $d$ for generating word $w$. Unsupervised topic adaptation can be achieved by interpolating a baseline model with a PLSA model as follows:

$$
p(w)=\lambda p_{\text {baseline }}(w)+(1-\lambda) p_{\text {PLSA }}(w)
$$

where $0<\lambda \leq 1$, the baseline model $p_{\text {baseline }}(w)$ usually is a standard higher order n-gram model enhanced with interpolation or back-off technologies [1][2].

\section{HIGHER ORDER MIXTURE COMPONENTS}

From Eq.(1) we know that PLSA models are unigram TMLMs in which mixture components are word unigram models. One simple way to extend the mixture components into higher order n-gram models is to perform $\mathrm{TD}$ to estimate the value of $p(t \mid d)$ and $p(w \mid t)$ first using the notion of mixture-of-unigrams. As $p(t \mid \boldsymbol{d})$ specifies the soft classification of the documents into latent topic classes, it can be directly used for building higher order topicspecific n-gram models. Given a classified document set $D=\{d \mid d=1,2, \cdots M\}$, for each of the topic $t$, we can build topic-specific n-gram models.

By averaging the n-gram counts with the topic probabilities of each document, n-gram model parameters can be estimated with ML approach which can be described as follows:

$$
p_{t}\left(w_{i} \mid w_{i-n+1}^{i-1}\right)=\frac{\sum_{d} C\left(w_{i-n+1}^{i}, d\right) p(t \mid d)}{\sum_{w} \sum_{d} C\left(w_{i-n+1}^{i-1} w, d\right) p(t \mid d)}
$$

where $C\left(w_{i-n+1}^{i}, d\right)$ represents the count of word sequence $w_{i-n+1} \ldots w_{i-1} w_{i}$ in document $d$ and $C\left(w_{i-n+1}^{i-1} w, d\right)$ carries the similar meaning.
Eq.(6) can be regarded as a generic training process of topic-specific LMs for which training documents are preclassified into topic domains. When hard classification method is used, documents are classified into disjoint classes, topic probability of a document is either 1 or 0 . The parameters of topic-specific n-gram model are estimated only from documents within the topic domain. When soft classification method is used, it discriminatively takes all the documents into consideration according to the probabilities of the documents falling into the topic classes. The parameters are trained from the average counts of ngrams of each document in the corpus.

Basically Eq.(6) is still a rough approximation, only the probabilities of the documents pertaining to the topic classes are used. This is equivalent to the assumption that words in documents are equally generated by topics, which actually is not the case in PLSA.

Knowing the topic $t$, the probability of word sequence $w_{1} \ldots w_{n}$ being generated by repeatedly using a single topic $t$ can be expressed as $p\left(w_{1} \cdots w_{n} \mid t\right)=\prod_{i=1}^{n} p\left(w_{i} \mid t\right)$. Вy taking this factor into consideration, Eq.(6) becomes:

$$
p_{t}\left(w_{i} \mid w_{i-n+1}^{j-1}\right)=\frac{p\left(w_{i} \mid t\right) \sum_{d} C\left(w_{i-n+1}^{j}, d\right) p(t \mid d)}{\sum_{w} p(w \mid t) \sum_{d} C\left(w_{i-n+1}^{j-1} w, d\right) p(t \mid d)}
$$

Eq.(7) is a refined training process, as opposed to Eq.(6). It uses both topic probabilities of the documents and the approximated probabilities of the n-grams being generated by the topic. As a result, in addition to weighting the training data at document level, the training process further narrows down the scope to individual n-gram units of each document in training corpus and weights the importance according to the relevance of the n-grams with the topic. The parameters of the n-gram models can also be regarded as being estimated from discounted average counts, which are obtained by discounting the average counts with the factors represented by the probabilities of n-grams being generated by the topic.

Both Eq.(6) and Eq.(7) can be regarded as the processes of manipulating n-gram counts of training corpus using unigram TMLMs.

\section{BAYESIAN TOPIC PREDICTION}

One of the important issues for applying TMLM is to predict topics of new documents, or to dynamically calculate the topic mixture weights from processing contexts. One approach for rapid topic prediction for a new document is to use Bayesian learning by which parameters can be updated based on new observations. Given a new document $d^{\prime}$, what we need to do is to estimate its topic probabilities $p\left(t \mid d^{\prime}\right)$ while topic-dependent word probabilities $p(w \mid t)$ are assumed to be consistent. In 
general, the MAP approach of Bayesian learning for parameter set $\theta$ from data set $d^{\prime}$ can be expressed as:

$$
\begin{aligned}
& \theta_{M A P}=\underset{\theta}{\arg \max } p\left(d^{\prime} \mid \theta\right) p(\theta) \\
& =\arg \max \left(\log p\left(d^{\prime} \mid \theta\right)+\log p(\theta)\right)
\end{aligned}
$$

In the context of our task $\theta=\left\{p\left(t \mid d^{\prime}\right)\right\}$ is assumed; $p\left(d^{\prime} \mid \theta\right)$ and $p(\theta)$ take the form of multinomial and Dirichlet distributions respectively; parameter estimation, being achieved by updating the expected counts of topic $t$ in $d^{\prime}$ from its initial value $\beta_{t, d^{\prime}{ }^{\prime}}$ to its true value $\beta_{t, d^{\prime}}$ [5][8], can be expressed as:

$$
\left\{\begin{array}{l}
\beta_{t, d^{\prime}}=\beta_{t, d^{\prime}}^{\prime}+\alpha \cdot \beta_{t, d^{\prime} 0} \\
\hat{p}\left(t \mid d^{\prime}\right)=\beta_{t, d^{\prime}} / \sum_{t} \beta_{t, d^{\prime}}
\end{array}\right.
$$

where $\alpha$ serves as a weighting factor of Bayesian learning between prior and current observations, $\beta_{t, d^{\prime}}^{\prime}$ is the expected count of topic $t$ appearing in $d^{\prime}$, it can be further elaborated by:

$$
\beta_{t, d^{\prime}}^{\prime}=\sum_{w} C\left(w, d^{\prime}\right) p\left(t \mid w, d^{\prime}\right)
$$

where item $p\left(t \mid w, d^{\prime}\right)$ in Eq.(9) represents the probability of generating $w$ from $d^{\prime}$ using topic $t$. The initial value of $\beta_{t, d^{\prime}}$ can be approximated by empirical calculation of the average topic counts of each document using worddocument table and TD results:

$$
\beta_{t, d^{\prime}{ }^{\prime}}=\sum_{w, d} C(w, d) p(t \mid d) / M=\bar{l} \cdot p(t)
$$

where $M$ is the number of documents in training corpus and $\bar{l}$ is the average document length in terms of number of words in the documents; topic prior probability $p(t)$ can be approximated from word-document counts in training corpus and topic probabilities of documents of TD results using $p(t)=\sum_{d} p(t, d)=\frac{\sum_{w, d} C(w, d) p(t \mid d)}{\sum_{w, d} C(w, d)}$.

From Eq.(8), (9) and (10), a close form solution for $\beta_{t, d^{\prime}}$ can be derived as:

$$
\beta_{t, d^{\prime}}=\sum_{w, d} C\left(w, d^{\prime}\right) p\left(t \mid w, d^{\prime}\right)+\alpha \bar{l} \cdot p(t)
$$

Eq.(11) represents the batch learning process in which document $d^{\prime}$ is expended once by $d^{\prime}$. Consequently, $\beta_{t, d^{\prime}}$, together with $p\left(t \mid d^{\prime}\right)$ are updated only once.

Another method called incremental learning can be easily realized by slight modification of the above process. Instead of adding $d^{\prime}$ to $d^{\prime}{ }_{0}$ once, the process can take place word by word. Starting from the initial topic counts, every time a word is added to $d^{\prime}{ }_{0}$, topic counts and posterior probabilities are changed according to the word added in. In this case $C\left(w, d^{\prime}\right)$ is always 1 in Eq.(10). The results estimated after adding the $(i-1)_{t h}$ word can be directly used as prior knowledge for updating these parameters after adding the $i_{t h}$ word, Eq.(11) becomes:

$$
\beta_{t, d_{i}^{\prime}}=p\left(t \mid w_{i}, d_{i-1}^{\prime}\right)+\alpha \cdot \beta_{t, d_{i-1}^{\prime}}
$$

where $p\left(t \mid d_{0}\right)=p(t)$. We experimented with both methods and found that incremental learning is working consistently better than batch learning with the tasks, it therefore is used in our experiments

\section{EXPERIMENTS}

We conduct experiments for perplexity tests on part of LDC corpus "English Gigaword LDC2005T12", which consists of units of documents distinguished by markup tags. The training data is selected from "nyt_eng" of year 2002 to 2004 of even and odd month alternatively, totaling $380 \mathrm{M}$ bytes disk space, 77,212 documents and 67.8 million words. Test data of 1000 documents are randomly selected from the remaining part.

\subsection{Topic Decomposition and Model Training}

The first step of building TMLMs is to perform topic decompositions. Some pre-processing works have to be carried out: stop-words around 600 in total, numerical expressions and words with unigram count less than 5 are removed. As a result a vocabulary of 135,956 words is selected for word-document table construction and topic prediction. Topic decompositions are performed on the word-document table using PLSA. After topic decomposition, topic dependent word n-gram models are built using Eq.(7). Separately, baseline n-gram models are built with standard method from the entire corpus. In particular, the 3-gram model contains total 6.183 million of probability entries. All the models employ the same vocabulary of $60 \mathrm{k}$ most frequent words in the training corpus; Witten-Bell back-off approach is used as the backoff strategy. 


\begin{tabular}{|l|c|c|c|c|c|c|c|c|}
\hline Topic & 0 & 1 & 2 & 3 & 4 & 5 & 6 & 7 \\
\hline $\mathrm{p}(\mathrm{t})$ & 0.056 & 0.103 & 0.127 & 0.135 & 0.19 & 0.173 & 0.073 & 0.143 \\
\hline \# of doc & 4023 & 9150 & 9322 & 10301 & 14007 & 13013 & 6375 & 11020 \\
\hline $\begin{array}{l}\text { \# of LM } \\
\text { entries }\end{array}$ & 1.113 & 1.978 & 2.339 & 2.347 & 3.192 & 2.912 & 1.335 & 2.825 \\
\hline
\end{tabular}

Table 1. Statistics of the results of topic decomposition component model training (8 topics)

Table 1 shows some statistics of the results of topic decomposition and component model training with the configuration of 8 topics and different document modeling approaches. The number of documents "\# of doc" in class $t$ means the average number of documents falling into the class, it is obtained by using $\sum_{d} p(t \mid d)$. The row of "\# of LM en-tries" represents the total number of probability entries in the models in million units.

\subsection{Performance against Baseline Models and Adaptation Approaches}

In our experiments, we compared the performances of baseline models with that of TMLMs implemented in different n-gram orders.

\begin{tabular}{|c|c|c|c|}
\hline Models & 1-gram & 2-gram & 3-gram \\
\hline Baseline & 1724.2 & 423.2 & 331.9 \\
\hline TMLM & 1509.3 & 362.2 & 285.8 \\
\hline
\end{tabular}

Table 2. Perplexity of baseline models and TMLMs built with 8 topics.

From Table 2 we notice that the performances of TMLMs are consistently better than those of baseline models. The performance of $n$-gram TMLM is comparable that of 3-gram baseline model. About $15 \%$ of perplexity reduction can be achieved on average by using TMLMs.

We compared the performances of TMLMs with those of LMs enhanced with unsupervised topic adaptation approaches indicated by Eq.(5). Table 3 summarizes the perplexity results of the models built with different number of topics. We observe from Table 3 that larger number of topics has positive influence on LM performances in general. Most importantly, TMLMs with small number of topics (for instance, 4) can substantially outperform LMs enhanced by unsupervised adaptation approaches in which lager number (for instance 20) of topics are used. With the same number of topics in the same document modeling paradigm, TMLM approach achieves overall $10 \%$ perplexity reduction over other adaptation approaches.

\begin{tabular}{|l|c|c|c|c|c|}
\hline \# of Topics & 4 & 8 & 12 & 16 & 20 \\
\hline Baseline+PLSA & 313.7 & 311.1 & 308.6 & 307.1 & 306.3 \\
\hline TMLM & 295.9 & 285.8 & 278.1 & 271.8 & 267.6 \\
\hline
\end{tabular}

Table 3. Perplexity results of adaptation approaches and TMLMs.
We investigated TMLM approaches by extending the mixture components of PLSA models from unigrams to higher order n-grams. A simple topic prediction method is proposed to facilitate the effort of estimating topic probabilities from new documents. We compared the performances TMLMs with those of standard n-gram models. We also compared TMLM approaches with unsupervised LM adaptation approaches. Perplexity test results have shown that $15 \%$ perplexity reduction can be achieved over baseline models, and around $10 \%$ perplexity reduction can be yielded over unsupervised approaches on average. TMLMs with smaller number of topics can still achieve better performances.

\section{REFERENCES}

[1] D. Gildea and T. Hofmann. 1999. Topic-based language models using EM, Proc. of Eurospeech.

[2] D. Mrva and P. C. Woodland. 2006. Unsupervised Language Model Adaptation for Mandarin Broad-cast Conversation Transcription, Proc. of ICSLP06.

[3] H-S. Chiu and B. Chen. 2007. Word Topical Mixture Models for Dynamic Language Model Adaptation, Proc. ICASSP'07, Honolulu, Hawai'I, U.S.A.

[4] J. Bellegarda, 2000. Exploiting Latent Semantic In formation in Statistical Language Modeling, IEEE Transaction on Speech and Audio Processing 88(80): 12791296.

[5] J.-T. Chien, M.-S. Wu and C.-S. Wu. 2005. Bayesian Learning for Latent Semantic Analysis, INTERSPEECH 2005, Lisbon, Portugal.

[6] K. Seymore, S. Chen, and R. Rosenfeld. 1998. Nonlinear interpolation of topic models for language model adaptation. In Proceedings of ICSLP98.

[7] R. M. Iyer and M. Ostendorf. 1999. Modeling Long Distance Dependence in Language: Topic Mixtures Versus Dynamic Cache Models, IEEE Trans. on Speech and Audio Processing, Vol. 7 No.1.

[8] S. Bai and H. Li. 2006. Bayesian Learning of N-Gram Statistical Language Modeling, ICASSP 2006.

[9] T. Hofmann. 1999. Probabilistic latent semantic indexing, 22nd ACM SIGIR Conference on Research and Development in IR, Berkeley, CA, USA

\section{CONCLUSIONS}

